\title{
P058. Refractory chronic migraine, fatigue and OnabotulinumtoxinA: a clinic setting experience
}

\author{
Filippo Baldacci , Martina Cafalli, Cinzia Lucchesi, Sonia Mazzucchi, Elisa Dini, Ubaldo Bonuccelli, Sara Gori \\ From Abstracts from the 1st Joint ANIRCEF-SISC Congress \\ Rome, Italy. 29-31 October 2015
}

\section{Objective}

To assess OnabotulinumtoxinA safety and efficacy in prophylactic treatment for chronic refractory migraine (headache occurring at least 15 days per month with lack of responsiveness to at least two preventive medications with established efficacy) [1] with associated fatigue symptom.

\section{Methods}

From March 2014 to May 2015 patients meeting the clinical diagnostic criteria for chronic refractory migraine were enrolled. Patients were treated with OnabotulinumtoxinA every three months according to the standard procedure (155-195 units) [2]. At baseline (T0) and after 6 months, at the third treatment (T1), a structured questionnaire was administered, including: a) migraine features [frequency (headache days/month), pain severity (Verbal Numeric Scale, VNS), acute medicines consumption/month, disability (Headache Impact Test, HIT-6), ictal cutaneous allodynia (Allodynia Symptoms Check-list 12, ASC-12)]; b) associated symptoms [fatigue (Fatigue Severity Scale, FSS), anxiety symptoms (Generalized Anxiety Disorder, GAD7), depressive symptoms (Patient Health Questionnaire, PHQ-9)]. Wilcoxon test was performed for the T0-T1 comparisons.

\section{Results}

Twenty-one patients were enrolled $(\mathrm{M} / \mathrm{F}=3 / 18$; mean age: 52.6 \pm 9.71$)$. A patient discontinued the study after the first treatment due to an adverse event (eyelid ptosis). Twenty patients were evaluated at $\mathrm{T} 1$, with migraine features changing as follow: T0 frequency $\mathrm{Me}=30 \mathrm{IQR}=10, \mathrm{~T} 1$ frequency $\mathrm{Me}=13 \mathrm{IQR}=13$, T0 VNS Me $=8 \mathrm{IQR}=3$, T1 VNS $\mathrm{Me}=8 \mathrm{IQR}=4$, T0 acute medicines consumption $/$ month
$\mathrm{Me}=20 \mathrm{IQR}=15, \mathrm{~T} 1$ acute medicines consumption/month $\mathrm{Me}=7 \mathrm{IQR}=13$, T0 HIT- $6 \mathrm{Me}=66 \mathrm{IQR}=7$, T1 HIT-6 $\mathrm{Me}=63 \mathrm{IQR}=13$, T0 ASC $-12 \mathrm{Me}=8 \mathrm{IQR}=5$, T1 ASC -12 $\mathrm{Me}=6 \mathrm{IQR}=7$. Associated symptoms changed as follow: $\mathrm{T0}$ FSS Me=48 IQR=19, T1 FSS Me=33 IQR=23, T0 GAD-7 $\mathrm{Me}=10 \mathrm{IQR}=8, \mathrm{~T} 1 \mathrm{GAD}-7 \mathrm{Me}=9 \mathrm{IQR}=7$, T0 PHQ-9 $\mathrm{Me}=10 \mathrm{IQR}=11, \mathrm{~T} 1 \mathrm{PHQ}-9 \mathrm{Me}=7 \mathrm{IQR}=7$. After two injection cycles with OnabotulinumtoxinA, a statistically significant reduction was found in: a) frequency $(\mathrm{p}=0.001 ; \mathrm{r}=$ $0.51) ; \mathrm{b})$ acute medicines consumption $/$ month $(\mathrm{p}=0.001$; $\mathrm{r}=0.54)$; $)$ FSS score $(\mathrm{p}=0.009 ; \mathrm{r}=0.41)$.

\section{Conclusions}

OnabotulinumtoxinA resulted well tolerated and effective in reducing not only frequency and acute medication use, but also fatigue in our population of chronic refractory migraineurs.

Written informed consent to publication was obtained from the patient(s).

Published: 28 September 2015

\section{References}

1. Schulman E: Refractory migraine-a review. Headache 2013, 53(4):599-61.

2. Aurora SK, Winner $P$, et al: OnabotulinumtoxinA for treatment of chronic migraine: pooled analyses of the 56-week PREEMPT clinical program. Headache 2011, 51(9):1358-73.

doi:10.1186/1129-2377-16-S1-A113

Cite this article as: Baldacci et al:: P058. Refractory chronic migraine,

fatigue and OnabotulinumtoxinA: a clinic setting experience. The Journal of Headache and Pain 2015 16(Suppl 1):A113.

\footnotetext{
* Correspondence: filippo.baldacci@unipi.it

Neurology Unit, Department of Clinical and Experimental Medicine,

University of Pisa, Pisa Italy
} 